\title{
Exame Clínico Objetivo Estruturado (ECOE): uma experiência de ensino por meio de simulação do atendimento farmacêutico
}

Dayani Galato ${ }^{1}$

Graziela Modolon Alano²

Tainã Formentin França ${ }^{3}$

Ana Cristina Vieira ${ }^{4}$

\section{Introdução}

A avaliação in loco de práticas clínicas é considerada como a mais fidedigna (Newble, 2000), no entanto, no processo educativo nem sempre é possível e, às vezes, apenas quando o futuro profissional de saúde está diante de um paciente real é que são identificados problemas relacionados às habilidades, atitudes e conhecimentos. Com o objetivo de realizar esta identificação antes da entrada no campo de estágio (Rutter, Hunt, 2003), ou mesmo de avaliar o desempenho de um profissional já formado (Fernandez, Franco, 2005; González et al., 2004; Weiss, 2004) é que foi desenvolvida a avaliação por simulação de atendimento.

Esta atividade é conhecida como Exame Clínico Objetivo Estruturado (ECOE), e tem sido aplicado, sobretudo, em escolas médicas (Carraccio, Englander, 2000). Portanto, o ECOE é uma ferramenta de medida de competências clínicas com a adoção de pacientes padronizados (Rushforth, 2007; Newble, 2004; Carraccio, Englander, 2000). Este exame pode ser filmado para facilitar o processo de avaliação (Humphris, Kaney, 2000). Instituir a simulação de atendimento na formação acadêmica significa incluir o enfoque problematizador, que, segundo Batista et al. (2005), auxilia na construção do conhecimento.

No Brasil, o ECOE foi utilizado pioneiramente pela faculdade de Medicina de Marília (Mazzoni, Moraes, 2006), e, na de Farmácia, foi inicialmente adotado durante uma gincana acadêmica nos anos 1990, em uma Universidade do Nordeste.

No curso de Farmácia da Universidade do Sul de Santa Catarina (Unisul) começou a ser adotado em 2003, como fruto de um projeto aprovado em 2002 pela Fundação de Apoio à Pesquisa do Estado de Santa Catarina (FAPESC), com o objetivo de construir um Programa de Desenvolvimento de Práticas Farmacêuticas (PDPFar).

Em julho desse mesmo ano, os integrantes do grupo de pesquisa foram convidados, pela Agência Nacional de Vigilância de Medicamentos (ANVISA) e pela Organização Pan-Americana da Saúde (OPAS), a realizarem um curso de capacitação para a prescrição racional. Durante a realização desse curso, os integrantes tiveram a experiência de passar por um ECOE de simulação de prescrição médica, padronizado pela Organização Mundial de Saúde, o que favoreceu o aprimoramento da simulação e o desenvolvimento de um instrumento de avaliação direcionado ao atendimento farmacêutico.

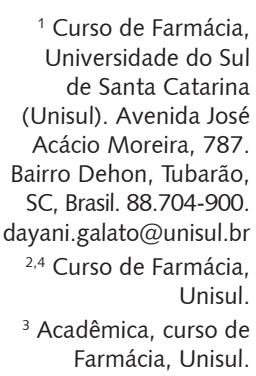


Posteriormente, o ECOE foi vinculado ao Estágio Supervisionado em Farmácia, que acontecia no último período curricular obrigatório. Sendo realizado ao final de um curso preparatório para o estágio. Nesse curso eram abordados conteúdos relativos à busca de informação, comunicação, processo de dispensação com prescrição e de automedicação responsável, além de um tutorial sobre o ECOE, onde era apresentado aos estudantes o instrumento de avaliação.

Esta atividade prévia à simulação de atendimento mostrou-se bastante adequada para reduzir a ansiedade dos acadêmicos ao ECOE e, ao mesmo tempo, é considerada pelos alunos como fundamental para a entrada no campo de estágio. Segundo os mesmos, transmite confiança e preenche lacunas advindas de um projeto pedagógico que não estava adequado às necessidades do profissional no mercado de trabalho. Cabe ressaltar que, a partir de 2007, foi implantado o currículo reformulado com base nas novas diretrizes curriculares (Leite et al., 2008), com estágios a partir do segundo ano de curso, no intuito de proporcionar ao acadêmico oportunidades de desenvolvimento de atitudes e habilidades em sua formação desde o início do curso. Neste contexto, este curso introdutório foi transformado em uma disciplina oferecida no segundo semestre do novo currículo.

O objetivo deste artigo, portanto, é apresentar a experiência de ensino no curso de Farmácia da Unisul no processo de ensino-aprendizagem por meio da simulação de atendimento farmacêutico (ECOE).

\section{Percurso metodológico}

Este trabalho refere-se a um relato de experiência com abordagem qualitativa (Minayo, 2004) da experiência educacional de simulação de atendimento farmacêutico vivenciada no curso de Farmácia da Unisul no período de 2005 a 2009. O processo foi desenvolvido por professores tutores da Disciplina de Estágio Supervisionado em Farmácia e aplicado aos acadêmicos do último período do curso.

O processo de simulação de atendimento farmacêutico foi construído em três etapas: a primeira relacionada com a preparação dos cenários e dos casos a serem simulados; a segunda, ao processo de filmagem, e, a terceira, ao processo de avaliação da simulação com aplicação do instrumento desenvolvido. Nos resultados deste artigo, essas etapas estão descritas de modo detalhado.

\section{A descrição do processo de simulação de atendimento farmacêutico e seus resultados}

\section{A primeira etapa: elaboração dos casos a serem simulados, padronização do paciente e preparação do cenário}

\section{Os casos simulados}

A definição dos problemas de saúde e dos medicamentos utilizados nas simulações de atendimento farmacêutico é realizada pela equipe de professores tutores do estágio, que selecionam os problemas de saúde mais prevalentes na região, e a lista de medicamentos usados para tratamento desses transtornos, os quais são repassados aos acadêmicos.

Os casos são elaborados com base na seleção anterior (problemas de saúde e medicamentos) e dividem-se em duas partes. A primeira é o relato do caso, onde o paciente (padronizado) repassa as informações essenciais, imediatamente ao iniciar sua conversa com o farmacêutico (acadêmico) e, a segunda parte, é composta por informações complementares, as quais o paciente expõe somente se for estimulado pelo farmacêutico por meio de indagações. Desta forma acredita-se que a simulação se aproxime da realidade enfrentada no atendimento farmacêutico, onde, na maioria das vezes, o paciente somente apresenta o motivo da procura ao Serviço solicitando um medicamento e, apenas se incentivado, fornece as informações sobre a situação clínica e as experiências com medicamentos.

A seguir, um exemplo de caso utilizado na simulação. 


\section{Descrição do caso}

Bruna, 35 anos, vai à farmácia, se aproxima do balcão e solicita uma medicação para tosse.

Informações complementares

- O medicamento não é para Bruna, mas para seu marido;

- Bruna é esposa de Jorge que tem 48 anos e está com tosse seca há três meses;

- Jorge não apresentou qualquer sintoma de gripe/resfriado recentemente;

- A tosse começou na mesma época em que houve alteração de seu tratamento medicamentoso da hipertensão, sendo prescrito, pelo médico, o captopril;

- A tosse incomoda muito o marido de Bruna, e, por isso, ela insiste em levar uma medicação para este problema.

\section{O paciente padronizado}

Inicialmente, os pacientes eram simulados por alunos voluntários das primeiras fases do curso de farmácia. No entanto, não foram observados bons resultados, pois, muitas vezes, os acadêmicos em processo de simulação, realizavam indagações referentes ao caso que não estavam contempladas nas informações complementares e, pelo fato de os voluntários não possuírem conhecimento suficiente do medicamento ou da doença em questão, acabavam por não responder aos questionamentos ou, mesmo, criavam respostas que confundiam os acadêmicos. A partir de 2006, a simulação dos pacientes passou a ser realizada por professores-padronizados e, com isso, foram evitados os problemas descritos anteriormente.

Outra situação vivenciada pelo grupo foi a caracterização dos pacientes simulados (roupas, maquiagem e outros acessórios), nem sempre fácil. Isto, às vezes, atrapalhava o atendimento feito pelo acadêmico devido à dificuldade do mesmo em reconhecer o paciente simulado como uma pessoa idosa, por exemplo. Uma estratégia criada para esta situação foi substituir o paciente idoso e criança, por um indivíduo adulto que vai à farmácia no lugar do paciente para adquirir o medicamento. Dessa forma, o paciente simulado representa o cuidador ou alguém que está realizando um auxílio.

\section{Montagem do cenário}

Inicialmente, o cenário foi montado em uma sala de aula com disponibilidade de mobília adquirida no projeto PDPFar (um balcão e prateleiras), além de diversas caixas vazias de medicamentos, entre eles, aqueles selecionados anteriormente. A organização dos medicamentos no armário era realizada por sistemas, para facilitar a localização dos mesmos pelo acadêmico. A organização por ordem alfabética de nome comercial ou por princípio ativo também poderiam ser adotadas, mas nunca foram testadas.

No cenário, ficavam, à disposição do acadêmico: telefone, calculadora, bloco de anotações, caneta, aparelho para aferição de pressão arterial e cadeira para realizar a aferição, além de bibliografia, como o Dicionário de Especialidades Farmacêuticas e a revista de preços de produtos comerciais. Nesse momento também se posicionava a câmera para a filmagem. Um detalhe a ser observado é que o acadêmico deve ser o principal sujeito da cena a ser filmada, assim, a câmera era posicionada para permitir a observação de detalhes, como a movimentação dos olhos.

Posteriormente, utilizou-se o espaço da Farmácia-Escola para a simulação, estando à disposição dos alunos toda a estrutura da mesma, incluindo medicamentos, sistema de gerenciamento informatizado, bibliografias, entre outros recursos.

\section{A segunda etapa: o processo de filmagem}

\section{Apresentação do cenário e filmagem da simulação}

A etapa de apresentação do cenário ocorre individualmente, cerca de cinco minutos antes da simulação. O professor tutor apresenta todo o cenário e coloca-se à disposição para o acadêmico esclarecer possíveis dúvidas sobre os medicamentos, a simulação e a utilização das fontes de informações disponíveis no cenário. 
Na simulação realizada na farmácia-escola, esta etapa de apresentação do cenário foi, na maioria das vezes, suprimida, pois os acadêmicos do estágio conhecem o cenário em questão e sua organização, sendo realizada esta etapa de apresentação apenas em situações pontuais.

O tempo de duração da filmagem depende do caso simulado e, também, do estilo de atendimento do acadêmico, levando em torno de três a sete minutos.

Uma vez concluída a filmagem, inicia-se a avaliação.

\section{A terceira etapa: O processo de avaliação e a descrição do instrumento desenvolvido}

\section{O processo de avaliação}

A sala preparada para essa etapa precisa ter um televisor e um aparelho de vídeo. Além disso, disponibiliza-se o material contendo a descrição completa do caso e o instrumento de avaliação. A avaliação é realizada de modo individual, porém pode ser observada por outros colegas da turma com prévio consentimento do acadêmico.

Abaixo são apresentadas as etapas da avaliação:

1 Leitura do caso: faz-se a leitura do caso incluindo as informações complementares;

2 Assiste-se a filmagem;

3 Faz-se uma breve discussão do caso;

4 Realiza-se a avaliação utilizando o instrumento de avaliação;

5 Calcula-se a nota a partir dos pesos estabelecidos para cada critério da ficha de avaliação;

6 É solicitada a assinatura do acadêmico no instrumento, juntamente com as assinaturas dos professores tutores.

A nota do atendimento era convertida em uma das notas da Disciplina de Estágio Supervisionado em Farmácia - no entanto, entendendo o processo educativo desta avaliação, por consenso entre os professores tutores, considerou-se que a participação do aluno nesta atividade seja a forma de conversão para a nota de estágio, e não mais o desempenho na simulação. Com esta alteração não se observou modificação no desempenho dos acadêmicos ao simularem, no entanto, percebeu-se mudança na postura dos mesmos frente às colocações dos professores tutores, ou seja, aumentou a receptividade dos acadêmicos às observações e às sugestões de aperfeiçoamento de algumas habilidades e atitudes na simulação de atendimento.

\section{A descrição do instrumento de avaliação}

O instrumento de avaliação está descrito no Quadro 1, sendo dividido em cinco etapas: definição do problema, indicação farmacêutica, informações e orientações ao paciente, estilo de comunicação e resolução do problema.

Alguns destes tópicos estão subdivididos em itens que auxiliam o avaliador a ter um olhar sobre todos os aspectos que necessitariam ser observados durante o processo de atendimento farmacêutico.

Para o cálculo da nota final, o primeiro critério, identificação do problema, corresponde a $20 \%$ da nota e é obtido pelo acadêmico ao receber conceito 4,0. Caso o acadêmico não alcance este conceito, é calculada a nota de acordo com o conceito equivalente. O segundo critério é a indicação farmacêutica, entendida, neste contexto, como automedicação responsável (World Health Organization, 1998) - somente será avaliada quando o caso simulado puder envolver a indicação de um tratamento medicamentoso, correspondendo a $20 \%$ da nota; do contrário, o terceiro critério, informações e orientações ao paciente, passará a valer $40 \%$ da nota ao invés de $20 \%$. O quarto critério, estilo de comunicação, corresponde a $20 \%$ da nota, e o último critério, resolução do problema, vale $20 \%$. 
Quadro 1. Instrumento de avaliação da simulação de atendimento farmacêutico

\section{Exame Clínico Objetivo Estruturado (ECOE) - Avaliação do professor tutor}

Farmacêutico (estagiário):

Avalie cada um dos critérios abaixo de acordo com os conceitos: 0 - não realizou; 1 - realizou de forma inadequada; 2 - realizou de forma incompleta; 3 - realizou bem; 4 - realizou muito bem; NA - não se aplica

1. Identificação do problema (20\%)

O farmacêutico identifica as necessidades do paciente e define claramente o problema que está tentando resolver $01234 \mathrm{NA}$

2. Indicação Farmacêutica (20\%)

O farmacêutico indica corretamente o tratamento ou fármaco (seleção adequada)

$01234 \mathrm{NA}$

Apresentação e posologia (forma farmacêutica, dose e intervalo de doses)

$01234 \mathrm{NA}$

Duração do tratamento

$01234 \mathrm{NA}$

Tratamento não medicamentoso

$01234 \mathrm{NA}$

3. Informações e orientações ao paciente (20 ou $40 \%$ )*

Efeitos positivos do medicamento (qual o efeito esperado, início, duração da ação)

$01234 \mathrm{NA}$

Instruções de uso (como tomar/administrar, frequência e intervalos de administração, quanto tempo utilizar, quais os cuidados com armazenamento)

$01234 \mathrm{NA}$

Advertências de uso (interações, contraindicações, dose máxima diária, efeitos adversos, quando e como suspender o tratamento)

01234 NA

Necessidade de seguimento dos resultados (o que fazer na sequência do tratamento)

$01234 \mathrm{NA}$

Orientações não farmacológicas

$01234 \mathrm{NA}$

*O percentual de $40 \%$ será utilizado nas situações em que não se aplica o processo de Indicação Farmacêutica no atendimento

4. Estilo de comunicação (20\%)

Fala de forma clara e compreensível (o tom/volume da voz é adequado, usa uma linguagem adequada ao nível de compreensão do paciente, usa paráfrases)

$01234 \mathrm{NA}$

Fala de forma coerente e sequencial

$01234 \mathrm{NA}$

Dá abertura a perguntas e expressões do paciente

$01234 \mathrm{NA}$

Assegura-se que o paciente compreende as instruções realizadas

$01234 \mathrm{NA}$

Comunicação não verbal

01234 NA

5. Resolução do problema (20\%)

O farmacêutico atendeu as necessidades e resolveu ou orientou para a resolução dos problemas do paciente.

$01234 \mathrm{NA}$

Avaliação:

Justificativas e/ou Comentários do avaliador:

Assinatura (avaliador):

Assinatura (aluno):

Data:

Data: 


\section{Discussão}

As Diretrizes Curriculares do Curso de Farmácia estabelecidas pelo Ministério da Educação (Brasil, 2002) preconizam uma formação generalista, humanista, crítica e reflexiva, e que o profissional seja competente para realizar os seus recursos cognitivos, afetivos e psicomotores diante da situação problema. Para tanto é fundamental, a uma Instituição de Ensino, o desenvolvimento do diálogo e da autoestima na relação professor-aluno, buscando o equilíbrio psicoemocional e ambiental do educando (Castro, 2004). Neste sentido, podem ser criados espaços de troca de experiências entre os professores tutores e os acadêmicos, buscando mais adequadamente a expressão da afetividade na relação com os pacientes.

Neste contexto, o ECOE auxilia o acadêmico a desenvolver habilidades necessárias ao atendimento clínico realizado na farmácia. Além do que, esta ferramenta auxilia na identificação de lacunas de conhecimento. Portanto, o ECOE constitui tanto uma ferramenta de avaliação quanto uma atividade educativa.

Segundo Troncon (2007), para a formação na área da saúde, constituem habilidades clínicas fundamentais: a comunicação, o exame físico, o raciocínio clínico e a proposição de medidas diagnósticas e terapêuticas. Para a realização das atividades clínicas farmacêuticas, como a dispensação e automedicação responsável, muitas destas habilidades também são destacadas (Galato et al., 2009, 2008).

Segundo Garcia (2001), o estágio realizado durante a graduação de cursos na área da saúde é fundamental, configurando-se a mais nobre ferramenta de ensino-aprendizagem. No entanto, o ECOE pode constituir uma alternativa complementar que pode ser adotada tanto na capacitação dos acadêmicos para o exercício profissional, quanto na avaliação desta atividade. Portanto, este exercício da prática, seja ele real ou simulado, é fundamental para a aquisição de proficiência nas habilidades clínicas (Troncon, 2007).

Na realização da atividade do ECOE, os docentes tutores devem estar envolvidos no Serviço de Saúde de forma que as situações criadas sejam as mais próximas do cotidiano. Pois, a educação do processo de cuidado se realiza cuidando e, segundo Garcia (2001, p.96), "educar é cuidar e para cuidar se educa". Para Noro e Noro (2002), quando o educador respeita a dignidade do acadêmico e trata-o com compreensão e ajuda construtiva, ele desenvolve a capacidade de procurar em si mesmo as respostas para seus problemas, tornando-o agente de seu próprio processo de aprendizagem.

Segundo Mazzoni e Moraes (2006), no ECOE, o acadêmico pode cometer equívocos sem prejudicar o paciente real, e o erro passa a ser visto como virtude, que pode ser modificada, e não penalizada. Uma vez identificado este erro, devem ser definidas estratégias para se evitar a recorrência do mesmo. A identificação destas limitações é fundamental na formação do profissional da saúde. Este benefício também é ressaltado por Troncon (2007), que destaca que o paciente simulado torna o aprendizado mais ativo, possibilitando homogeneidade nas oportunidades de treinamento e repetições, facilitando as correções nas ações realizadas.

Segundo Galato et al. (2008), a prática clínica farmacêutica é aperfeiçoada pelo processo de ação-reflexão-ação. Este processo é facilitado no ambiente do ECOE, visto que, segundo Troncon (2007), entre as principais estratégias que envolvem o aprendizado das habilidades clínicas, estão: o desenvolvimento do conhecimento por meio de leituras, aulas e demonstrações; a prática inicial com paciente; os comentários do observador (professor tutor); a reflexão, seguida de novas ações, avaliações e reflexões.

Neste contexto, um dos aspectos que pode ser analisado com bastante propriedade no ECOE é a comunicação, em especial a não verbal, por envolver uma etapa de filmagem (Humphris, Kaney, 2000). A comunicação pode ser verbal e não verbal (Silva, 1993). A primeira expressa o ser social e, a segunda, o ser psicológico (Silva et al., 2000; Silva, 1993).

O conhecimento da linguagem corporal pode melhorar o cuidado em saúde, uma vez que possibilita a ampliação da percepção profissional (Weil, Tompakow, 2004; Silva et al., 2000). Assim, quando se aborda o tema comunicação, destaca-se que, entre os componentes essenciais para o desenvolvimento da relação terapêutica, está a empatia (Cipolle, Linda, Morley, 2000). Desta forma, é importante que o profissional de 
saúde controle seus sentimentos e emoções, mas sem desumanizar o Serviço.

A comunicação durante a simulação de atendimento sofre a influência direta do grau de conhecimento do acadêmico. Isto se reflete na forma como é realizada a coleta de dados do paciente (identificação do problema), bem como no repasse de informações.

Cabe ressaltar que, segundo Troncon (2007), quando se realiza a simulação de atendimento utilizando-se pacientes padronizados, existe a possibilidade de se terem algumas desvantagens, que, no caso da adoção do professor tutor como paciente padronizado, como realizado nesta experiência, podem ser, em parte, amenizadas. Por outro lado, a adoção de professores pode inibir a atuação do acadêmico no atendimento clínico. Porém, quando o professor envolvido nessa atividade mantém uma relação igualitária, demonstrando habilidades de criticar e elogiar com estímulo, facilita o processo de ensino-aprendizagem (Nuto et al., 2005).

No atendimento clínico farmacêutico, é possível que o profissional busque informações, e esta busca pode ocorrer durante o atendimento, em especial em literaturas disponibilizadas no próprio serviço, bem como, em outro momento, na ausência do paciente. As informações disponíveis no ambiente da farmácia, na sua grande maioria, caracterizam-se como sendo comerciais. Franceschet e Farias (2005) demonstraram que o Dicionário de Especialidades Farmacêuticas foi descrito como principal fonte de informação por $95,6 \%$ dos farmacêuticos entrevistados. Tentando reproduzir este contexto no cenário do ECOE, são disponibilizadas fontes de informação comercial. Destaca-se que a busca de informação e a atualização profissional é uma atitude requerida pelos pacientes que procuram o estabelecimento farmacêutico com a visão de um estabelecimento de saúde, e não apenas um comércio de medicamentos (Galato, Angeloni, 2009).

Um resultado importante deste trabalho foi o instrumento para a avaliação da simulação de atendimento farmacêutico. Destaca-se que o mesmo foi desenvolvido com base no modelo apresentado pela Organização Mundial da Saúde (Organização Mundial da Saúde, 2001). Considerando-se alguns dos fundamentos do atendimento clínico farmacêutico (Galato et al., 2009, 2008), o ECOE foi adaptado pelos professores tutores, estruturando-se de forma a possibilitar a avaliação desse processo de atendimento.

Os resultados obtidos com a aplicação do instrumento possibilitam a identificação dos erros, que, muitas vezes, podem refletir lacunas de conhecimento ou falta de habilidades. Estes pontos auxiliaram os professores tutores no processo de ensino-aprendizagem (Garcia, 2001), bem como serviram, no curso de Farmácia/ Unisul, como indicadores para a elaboração do novo currículo pedagógico voltado às novas diretrizes curriculares, inserindo, desta forma, várias atividades simuladas desde o início do curso.

Como já mencionado anteriormente, a nota resultante da aplicação desse instrumento (desempenho) deixou de ser convertida para o Estágio Supervisionado em Farmácia, observandose maior receptividade dos acadêmicos às sugestões de aperfeiçoamento de habilidades e atitudes na simulação de atendimento. No estudo de Nuto et al. (2005) com estudantes da área da saúde, ficou evidente que a preocupação maior dos alunos é com algum erro no atendimento ao paciente, e, consequentemente, com a avaliação que o professor fará, ficando o bem-estar do paciente em segundo plano. Dessa forma, o fato de o ECOE não estar centrado na produção de uma nota, além de facilitar as intervenções do professor tutor no sentido de aprimoramento do atendimento, permite ao acadêmico deixar de lado a ansiedade e focar sua atenção na melhoria de suas habilidades.

O curso preparatório do estágio feito antes dessa atividade contribui para reduzir a ansiedade dos alunos ao ECOE e trazer autoconfiança. O aumento da autoconfiança melhora seu desempenho técnico e humano, de acordo com Nuto et al. (2005). Os processos emocionais na aprendizagem geram as mudanças cognitivas. Assim, os sentimentos de ansiedade, tensão, curiosidade, entusiasmo, frustração, alegria, impaciência, obstinação, surgidos no processo ensino-aprendizagem, são importantes e acompanham o ato de perceber, analisar, comparar e entender (Bordenave, Pereira, 2002). Os professores tutores do ECOE identificam que, tanto os acertos quanto os erros ou limitações apresentadas pelo acadêmico durante o atendimento costumam ficar na sua memória, pois trata-se de uma experiência bastante intensa e, portanto, o conduz ao aprendizado. 


\section{Considerações finais}

O processo aqui descrito apresenta a aplicação e adaptação desta ferramenta pedagógica na formação acadêmica, voltada ao desenvolvimento de habilidades clínicas fundamentais aos profissionais da saúde. Desta forma, a simulação de atendimento farmacêutico possibilita ao acadêmico uma oportunidade de melhorar as suas habilidades e atitudes na prestação dos serviços clínicos farmacêuticos e na educação em saúde do paciente, além de aprofundar os conhecimentos a respeito das situações simuladas por meio do processo ação-reflexão-ação.

O ECOE contribui para o desenvolvimento do equilíbrio psicoemocional e ambiental do educando, a fim de reduzir suas ansiedades acerca da aplicação, na prática, dos conhecimentos abordados na universidade, levando-o a adquirir autoconfiança e melhorando, em especial, os aspectos relacionados à comunicação com o paciente.

Destaca-se que o uso de pacientes padronizados em situações simuladas favorece, em especial, a padronização do processo de ensino-aprendizagem, bem como reduz os possíveis equívocos que possam a vir a causar dano ao paciente real.

Os principais desafios para a adoção desta ferramenta de ensino estão relacionados ao perfil adequado do corpo docente e à capacidade de formulação dos problemas clínicos a serem trabalhados. No entanto, uma vez superados estes desafios, esta ferramenta de ensino permite uma formação clínica dos farmacêuticos por intermédio da articulação dos conhecimentos teóricos aplicados à resolução de problemas clínicos reais.

Neste contexto, o ECOE é visualizado como uma ferramenta que auxilia na formação dos profissionais da saúde em consonância com as novas Diretrizes Curriculares, e que pode ser facilmente reproduzido em outras instituições de ensino.

\section{Colaboradores}

As autoras Dayani Galato e Graziela Modolon Alano trabalharam juntas em todas as etapas do manuscrito e do trabalho experimental. Tainã Formentin França atuou na elaboração do manuscrito e Ana Cristina Vieira esteve envolvida no trabalho experimental e revisão do manuscrito.

\section{Agradecimentos}

Agradecemos a todos que contribuíram no aprimoramento desta ferramenta de ensinoaprendizagem, em especial, ao professor Wellington Barros da Silva.

\section{Referências}

BATISTA, N. et al. O enfoque problematizador na formação de profissionais da saúde. Rev. Saude Publica, v.39, n.2, p.231-7, 2005.

BORDENAVE, J.E.D.; PEREIRA, A.M. Estratégias de ensino-aprendizagem. 23.ed. Petrópolis: Vozes, 2002.

BRASIL. Conselho Nacional de Educação. Resolução CNE/CES 2, de 19 de fevereiro de 2002. Institui as Diretrizes Curriculares Nacionais do Curso de Graduação em Farmácia. Disponível em: <http://www.portal portal. mec.gov.br/cne/arquivos/pdf/CES022002. pdf>. Acesso em: 29 ago. 2009.

CARRACCIO, C.; ENGLANDER, R. The objective structured clinical examination: a step in the direction of competency-based evaluation. Arch. Pediatr. Adolesc. Med., v.154, n.7, p.736- 41, 2000. 
CASTRO, F.C. Os temores na formação e prática da medicina: aspectos psicológicos. Rev. Bras. Educ. Med., v.28, n.1, p.38-45, 2004.

CIPOLLE, R.J.; LINDA; M.S.; MORLEY, P.C. El ejercicio de la atención farmacêutica. Madrid: McGraw-Hill, 2000.

FERNANDEZ, J.L.T.; FRANCO, B.P. Examen de ingenios para lãs ciências: Edipalaci frente a ECOE. Aten. Primaria, v.35, n.5, p.273, 2005.

FRANCESCHET, I.S.; FARIAS, M.R. Investigação do perfil dos farmacêuticos e das atividades desenvolvidas em farmácias do setor privado no município de Florianópolis, Santa Catarina, Brasil. Acta Farm. Bonaer., v.24, n.4, p.590-7, 2005.

GALATO, D.; ANGELONI, L. A farmácia como estabelecimento de saúde: a visão do usuário de medicamentos. Rev. Bras. Farm., v.90, n.1, p.14-8, 2009.

GALATO, D. et al. A dispensação de medicamentos: uma reflexão sobre o processo para prevenção, identificação e resolução de problemas relacionados com a farmacoterapia. Rev. Bras. Cienc. Farm., v.44, n.3, p.465-75, 2008.

. Responsible self-medication: a reflection on the process of pharmaceutical intervention: review of the process of pharmaceutical attendance. Braz. J. Pharm. Sci., v.49, n.4, p.625-33, 2009.

GARCIA, M.A.A. Saber, agir e educar: o ensino-aprendizagem em serviços de saúde. Interface - Comunic., Saude, Educ., v.5, n.8, p.89-100, 2001.

GONZÁLEZ, M.P. et al. Evaluación de la competencia clínica de tutores de residentes de medicina familiar y comunitaria. Aten. Primaria, v.34, n.2, p.68-74, 2004.

HUMPHRIS, G.M.; KANEY, S. The objective structured video exam for assessment of communication skills. Med. Educ., v.34, n.11, p.939-45, 2000.

LEITE, S.N. et al. I Fórum Nacional de Educação Farmacêutica: o farmacêutico que o Brasil necessita. Interface - Comunic., Saude, Educ., v.12, n.25, p.461, 2008.

MAZZONI, C.J.; MORAES, M.A.A. A avaliação prática estruturada de habilidades clínicas na Famema: fundamentos para construção e aplicação. Gestão Univ. jun. 2006. Disponível em: <http://www.gestaouniversitaria.com. php/edicoes/68-99/321-aavaliacao-pratica-estruturada-de-habilidades-clinicas-na-famema--fundamentos-paraconstrucao-e-aplicacao.html>. Acesso em: 22 set. 2009.

MINAYO, M.C.S. O desafio do conhecimento: pesquisa qualitativa em saúde. 8.ed. São Paulo: Hucitec, 2004.

NEWBLE, D. Techniques for measuring clinical competence: objective structured clinical examination. Med. Educ., v.38, n.2, p.199-203, 2004.

NEWBLE, D.I. Assessment of clinical competence. BJA, v.84, n.4, p.432-3, 2000.

NORO, S.E.M.; NORO, L.R.A. A auto-estima como facilitador do processo ensinoaprendizagem. Rev. Humanidades, v.17, n.2, p.113-9, 2002.

NUTO, S.A.S. et al. O processo ensino-aprendizagem e suas conseqüências na relação professor-aluno-paciente. Cienc. Saude Colet., v.11, n.1, p.89-96, 2006.

ORGANIZAÇÃO MUNDIAL DA SAÚDE. Guia do instrutor em práticas da boa prescrição médica. 2001. Disponível em: <http://www.opas.org.br/medicamentos/ temas_documentos_detalhe.cfm?id=43\&iddoc=241>. Acesso em: 22 set. 2009 .

RUSHFORTH, H.E. Objective structured clinical examination (OSCE): review of literature and implications for nursing education. Nurse Educ. Today, v.27, n.5, p. 481-90, 2007.

RUTTER, P.M.; HUNT, A. The development of a managed learning environment using WedCT to faciliate 4 th year M. Pharm undergraduates ability to counsel patients en preparation for OSCEs. Pharm. Educ., v.3, n.1, p.63-6, 2003. 
SILVA, L.M.G. et al. Comunicação não-verbal: reflexões acerca da linguagem corporal. Rev. Latino-am. Enferm., v.8, n.4, p.52-8, 2000.

SILVA, M.J.P. Construção e validação de um programa sobre comunicação não-verbal para enfermeiros. 1993. Tese (Doutorado) - Escola de Enfermagem, Universidade de São Paulo, São Paulo. 1993.

TRONCON, L.E.A. Utilização de pacientes simulados no ensino e na avaliação de habilidades clínicas. Medicina (Ribeirão Preto), v.40, n.2, p.180-91, 2007.

WEIL, P.; TOMPAKOW, R. O corpo fala: a linguagem silenciosa da comunicação não verbal. 57.ed. Petrópolis: Vozes, 2004.

WEISS, B.D. Are we competent to assess competence? Fam. Med., v.36, n.3, p.214-6, 2004.

WORLD HEALTH ORGANIZATION. The role of the pharmacist in self-care and selfmedication. The Hangue: World Health Organization, 1998. Disponível em: <www.who. int/medicinedocs/pdf/whozip32e/whozip32e.pdf>. Acesso em: 17 set. 2009. 
A simulação de atendimento farmacêutico tem por finalidade avaliar as competências clínicas dos acadêmicos. Constitui uma ferramenta pedagógica que possui três etapas: a) preparação do cenário e dos casos; b) simulação; e c) avaliação. Os casos simulados consistem de informações essenciais que são fornecidas espontaneamente ao acadêmico no início da simulação e outras complementares, que somente tornar-se-ão conhecidas se forem investigadas. Toda a simulação de atendimento é filmada, o que permite a análise da comunicação. O processo de avaliação é iniciado com a leitura do caso, a análise do vídeo e a aplicação do instrumento desenvolvido. O ECOE possibilita ao acadêmico uma oportunidade de melhorar as suas habilidades e atitudes na prestação do atendimento farmacêutico, além de aprofundar os conhecimentos a respeito das situações simuladas.

Palavras-chave: Formação de profissionais da saúde. ECOE. Simulação. Metodologia de ensino. Educação em farmácia. Atendimento farmacêutico.

\section{Objective structured clinical examinations (OSCE): a teaching experience using simulation of pharmaceutical care}

Simulation of pharmaceutical care aims to assess students' clinical skills. It comprises an educational tool that has three steps: (a) setting the stage and preparing the cases; (b) simulation; and (c) evaluation. The simulated cases consist of information that is provided spontaneously to students at the beginning of the simulation and other, complementary information that only becomes known if it is investigated. All of the simulated attendance is filmed, which enables analysis of the communication. The evaluation process starts with reading the case, analysis of the video and application of the tool that has been developed. This examination provides students with an opportunity to improve their skills and attitudes in relation to provision of pharmaceutical care, in addition to deepening their knowledge about the situations that were simulated.

Keywords: Training for healthcare professionals. OSCE. Simulation. Teaching methodology. Pharmacy education. Pharmaceutical care.

Examen clínico, objetivo estructurado (ECOE): una experiencia de enseñanza por medio simulación de la atención del farmacéutica

La simulación de atención farmacéutica tiene por finalidad la de evaluar las capacidades clínicas de los académicos. Constituye en una herramienta pedagógica que posee tres etapas: a) preparación del lugar y de los casos, b) simulación; y c) evaluación. Los casos simulados constan de informaciones esenciales proporcionadas espontáneamente al académico en el inicio de la simulación y otras complementarias que sólo se conocerán si son investigadas. Se filma toda la simulación de atención, lo que permite el análisis de la comunicación. El proceso de evaluación se inicia con la lectura del caso, el análisis del vídeo y la aplicación del instrumento desarrollado. El ECOE posibilita al académico una oportunidad de mejorar sus habilidades y actitudes en la prestación de la atención del farmacéutica, además de profundizar los conocimientos respecto a las situaciones simuladas.

Palabras clave: Formación de profesionales de la salud. ECOE. Simulación. Metodología de enseñanza. Educación en farmacia. Atención farmacêutica. 
